\title{
The Survival of Head and Neck Carcinoma Patients Depends on Secondary Causes of Death
}

\author{
André Buchali1,2, Winfried Huhnt'1, Ina Maekelburg1, Sophia Rohrberg1, Dietrich Sidow ${ }^{1}$, \\ Eyck Blank ${ }^{1}$, Christina Schroeder ${ }^{3}$, Achim Franzen ${ }^{2,4}$
}

${ }^{1}$ Department of Radiooncology, Ruppiner Kliniken, Neuruppin, Germany

${ }^{2}$ Medical University Theodor Fontane, Neuruppin, Germany

${ }^{3}$ Justus Liebig University, Giessen, Germany

${ }^{4}$ Department of Otorhinolaryngology Head and Neck Surgery, Ruppiner Kliniken, Neuruppin, Germany

Email: a.buchali@ruppiner-kliniken.de

How to cite this paper: Buchali, A., Huhnt, W., Maekelburg, I., Rohrberg, S., Sidow, D., Blank, E., Schroeder, C. and Franzen, A. (2017) The Survival of Head and Neck Carcinoma Patients Depends on Secondary Causes of Death. Journal of Cancer Therapy, 8, 1187-1193.

https://doi.org/10.4236/jct.2017.813102

Received: November 18, 2017

Accepted: December 11, 2017

Published: December 14, 2017

Copyright $\odot 2017$ by authors and Scientific Research Publishing Inc. This work is licensed under the Creative Commons Attribution-NonCommercial International License (CC BY-NC 4.0). http://creativecommons.org/licenses/by-nc/4.0/ (c) (7) (8) Open Access

\begin{abstract}
Purpose: The study aimed to analyse causes of death and differential survival after multimodal treatment of head and neck carcinoma patients. Methods and Materials: Between September 2000 and December 2015, 935 patients received a multimodal treatment of head and neck carcinoma. Of these, $562 \mathrm{pa}$ tients (60.1\%) underwent a resection of the primary tumour and a postoperative radio- or radiochemotherapy and 373 patients (39.9\%) received definitive radio- or radiochemotherapy. The median follow-up was 21.0 months (0.5 175.4 months). Results: At the endpoint of the investigation, 465 patients (49.7\%) were alive. The median survival of all patients was 44.8 months (0.5 164.3 months). A total of 470 patients $(50.3 \%)$ died. The causes of death were divided into five groups: 22 patients $(2.4 \%)$ died of therapy-associated complications with a median of 2.2 months (1.6 - 3.3 months). The 160 patients (17.1\%) with intercurrent death, 117 patients (12.5\%) with deaths from locoregional progression with or without metastasis, and 86 patients $(9.2 \%)$ with deaths from metastasis without locoregional recurrence showed comparable survival curves with a median survival of 13.3 months, 13.6 months, and 14.4 months. Eighty-five patients (9.1\%) died from second malignant diseases and controlled treated head and neck carcinomas with a median survival of 34.5 months $(P<0.001)$. Conclusion: Despite a locoregional control and metastasis-free survival of $78.3 \%$ patients, only $49.7 \%$ of the patients were alive, due to a high rate of intercurrent and second malignant diseases. The short median survival rate is mainly due to the tumours (locoregional and or distant progression) and intercurrent causes of death, with the second malignant diseases leading to death later on.
\end{abstract}




\section{Keywords}

Head and Neck Carcinoma, Radiochemotherapy, Causes of Death

\section{Introduction}

Multimodal treatment of patients with head and neck carcinoma results in disappointing overall survival rates, despite the substantially better disease-free survival rates in definitive as well as postoperative radiochemotherapy trials. An increased comorbidity of these patients, especially due to smoking and alcohol consumption, is discussed. We analysed our head and neck carcinoma patients multimodally treated with a curative intention from September 2000 to December 2015 .

The purpose was to analyse the causes of death and their influence on survival.

\section{Methods and Materials}

Between September 2000 and December 2015, 935 patients (772 male, 163 female) received a multimodal treatment of head and neck carcinoma. The mean age of the patients was 59.3 years (30.5 - 90.2 years). Of these, 562 patients (60.1\%) underwent a resection of the primary tumour and a postoperative radioor radiochemotherapy and 373 patients (39.9\%) received definitive radio- or radiochemotherapy only. After treatment, all patients underwent frequent follow-up examinations with an otolaryngologist and a radiooncologist. The median follow-up was 21.0 months (0.5 - 175.4 months), and the loss to follow-up was $13.3 \%$ (124 patients). Patients' characteristics are shown in Table 1. A total of 832 patients $(89 \%)$ received the treatment primarily, 103 patients (11\%) had a recurrence after a history of an initially resected small primary lesion without adjuvant treatment and were multimodal treated at the time of locoregional recurrence without distant metastasis. Sixty-three of the patients (6.7\%) completed their irradiation in an untimely manner.

Survival rates were estimated according to the Kaplan-Meier method; differences between groups were assumed by means of the log-rank statistic. For statistical analysis, Microsoft Excel 2008 and SPSS Version 23 were used.

\section{Results}

At the endpoint of the investigation, 465 patients $(49.7 \%)$ were alive. The median survival of all patients was 44.8 months (0.5 - 164.3 months). During the investigated interval, 470 patients (50.3\%) died. Female patients showed a median survival of 80.3 months whereas male patients had a median survival of 40.3 months $(P<0.001)$ (Figure 1$)$. While patients with postoperative radio(chemo-)therapy had a median survival of 65.2 months, patients with a definitive treatment had a significantly shorter median survival of 21.7 months $(P<$ 0.001) (Figure 2). 
Table 1. Patient characteristics.

\begin{tabular}{|c|c|c|}
\hline Gender & & \\
\hline Male & 772 & $82.6 \%$ \\
\hline Female & 163 & $17.4 \%$ \\
\hline \multicolumn{3}{|c|}{ Primary Tumour Localisations } \\
\hline Oropharynx & 340 & $36.4 \%$ \\
\hline Larynx & 140 & $15.0 \%$ \\
\hline Hypopharynx & 131 & $14.0 \%$ \\
\hline Floor of the mouth & 120 & $12.8 \%$ \\
\hline Tongue & 53 & $5.7 \%$ \\
\hline $\mathrm{CuP}$ & 48 & $5.1 \%$ \\
\hline Nasopharynx & 39 & $4.2 \%$ \\
\hline Paranasal sinuses & 25 & $2.7 \%$ \\
\hline Salivary glands & 19 & $2.0 \%$ \\
\hline Skin & 11 & $1.2 \%$ \\
\hline Ear canal & 5 & $0.5 \%$ \\
\hline Lip & 4 & $0.4 \%$ \\
\hline \multicolumn{3}{|l|}{ UICC Stage } \\
\hline I & 31 & $3.3 \%$ \\
\hline II & 72 & $7.7 \%$ \\
\hline III & 129 & $13.8 \%$ \\
\hline IVA & 656 & $70.2 \%$ \\
\hline IVB & 46 & $4.9 \%$ \\
\hline IVC & 1 & $0.1 \%$ \\
\hline \multicolumn{3}{|c|}{ Primary Tumour Resection } \\
\hline Without resection & 373 & $39.9 \%$ \\
\hline R0 & 381 & $40.7 \%$ \\
\hline R1 & 181 & $19.4 \%$ \\
\hline
\end{tabular}

The causes of death were divided into five groups: therapeutic-associated deaths, intercurrent deaths with locoregional and systemic tumour control, deaths due to and after a locoregional recurrence, deaths with distant metastasis and a locoregional controlled tumour, and deaths from a second malignant disease. Twenty-two patients (2.4\%) died from therapy-associated complications after a median of 2.2 months (1.6 - 3.3 months) and had a significant lower median survival (Table 2, Figure 3).

The 160 patients (17.1\%) with intercurrent deaths, 117 patients $(12.5 \%)$ with deaths from locoregional progression with or without metastasis, and 86 patients (9.2\%) with deaths from metastasis without locoregional recurrence showed comparable survival curves with a median survival of 13.3 months, 13.6 months, 


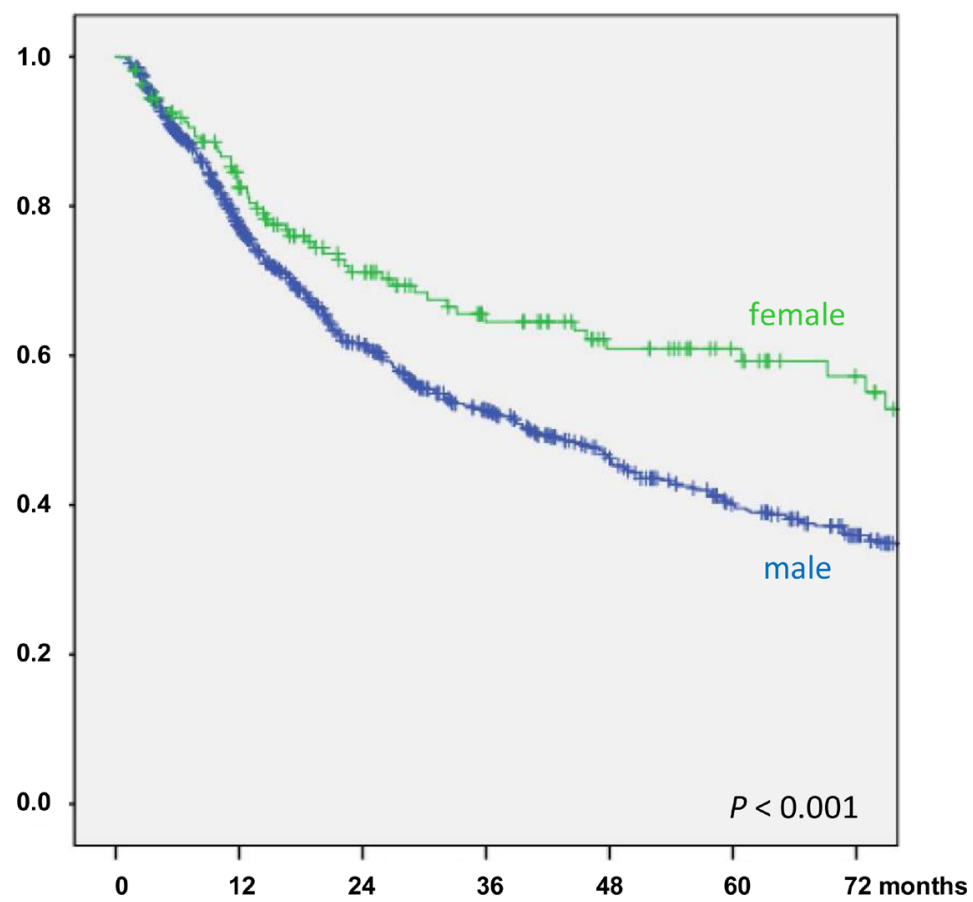

Figure 1. Survival rates (months) of male and female patients.

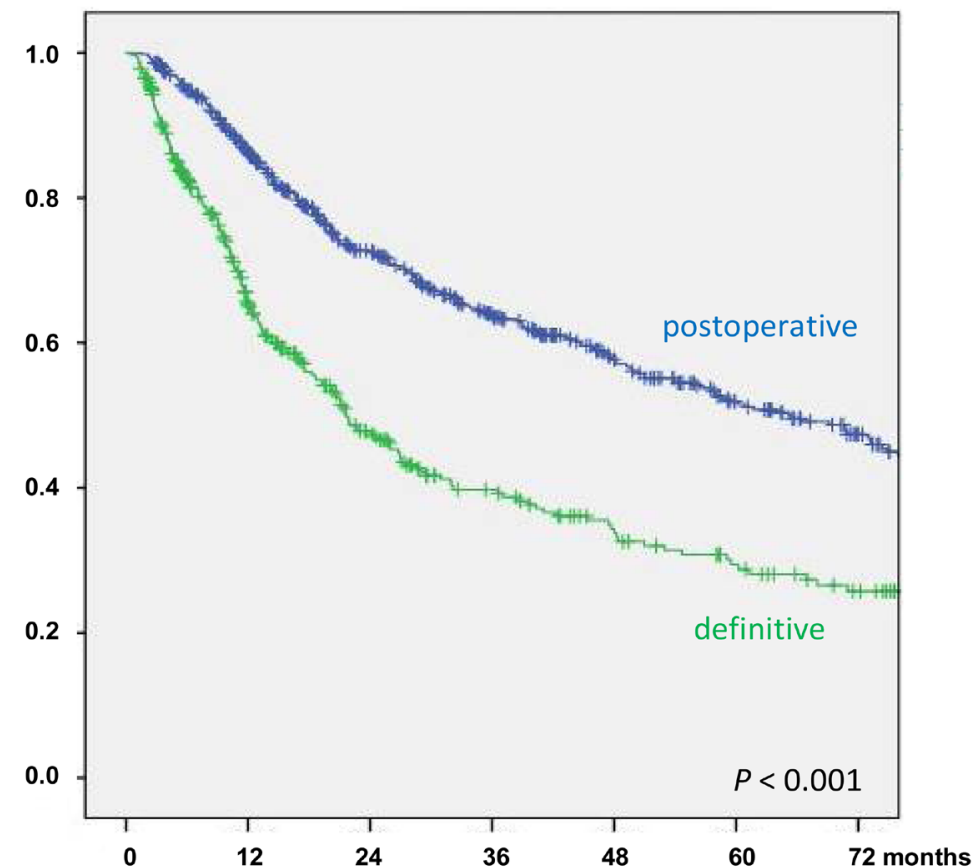

Figure 2. Survival rates (months) of postoperative and definitive treated patients.

and 14.4 months, respectively. Eighty-five patients $(9.1 \%)$ died from a second malignancy and controlled head and neck carcinoma. They had a significantly longer median survival of 34.5 months $(P<0.001)$. Fifty-one of the $85(60 \%) \mathrm{fa}$ tal second malignant diseases were lung cancer diseases, 10 patients $(12 \%)$ 


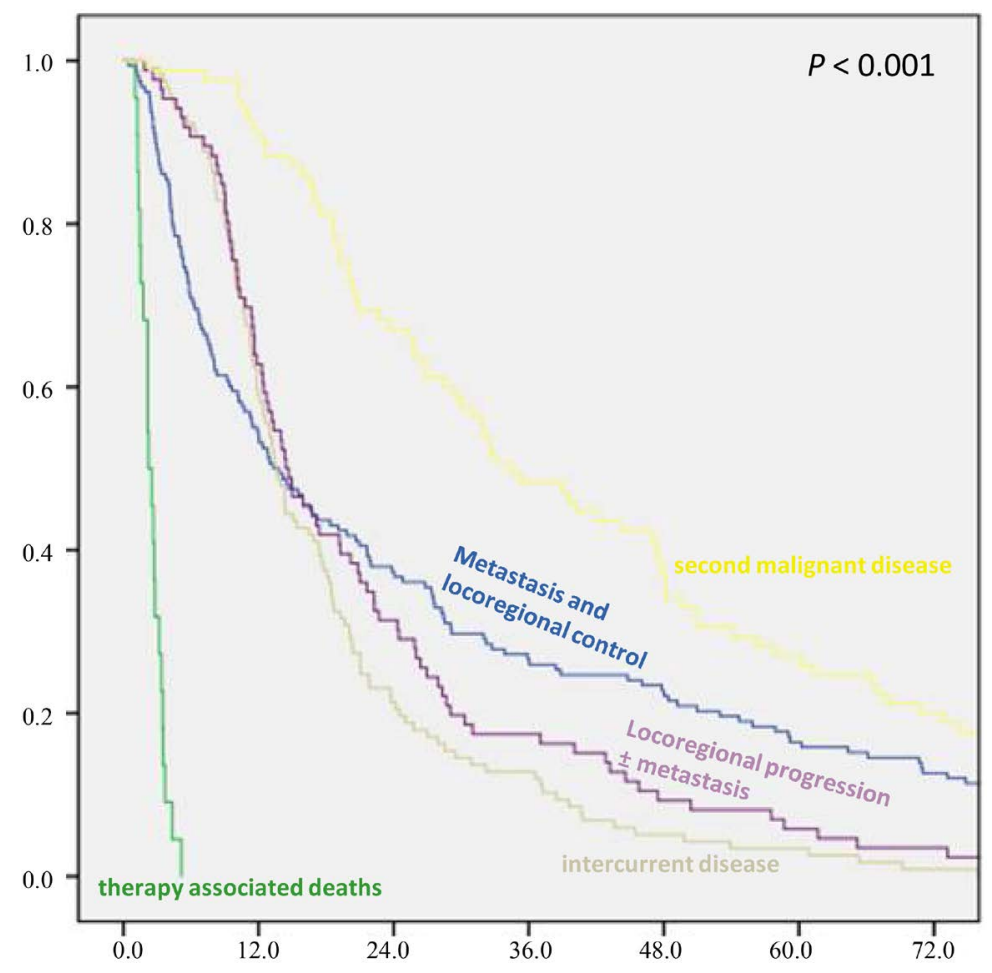

Figure 3. Survival rates depending on the cause of death (green line: therapy-related death, grey: locoregional progression, purple: distant metastasis locoregional control, blue line: intercurrent disease, yellow line: second malignant disease).

Table 2. Survival of patients depending on causes of death.

\begin{tabular}{cccc}
\hline & $\mathrm{n}$ & $(\%)$ & Median Survival \\
\hline All patients & 935 & 100 & 16.0 months \\
Deceased patients & & & \\
Therapy associated & 22 & 4.7 & 2.4 months \\
Intercurrent disease & 160 & 34.0 & 13.2 months \\
Locoregional progression \pm metastasis & 117 & 24.9 & 13.6 months \\
Metastasis and locoregional control & 86 & 18.3 & 14.6 months \\
Second malignant disease & 85 & 18.1 & 34.5 months \\
\hline
\end{tabular}

suffered from oesophageal carcinoma, and 5 patients (6\%) from a second head and neck carcinoma.

\section{Discussion}

\subsection{Locoregional Control Rates}

For patients receiving postoperative radio- or radiochemotherapy, locoregional control rates after 2 years were between $46 \%$ and $82 \%$ [1] [2] and after 5 years were between $36 \%$ and $80 \%$ [1] [2]. In comparison to this, our patients had lo- 
coregional control rates after 2 and 5 years of $86.1 \%$ and $80.8 \%$, respectively. The published data of locoregional control rates after definitive radio- or radiochemotherapy after 2 years, 3 years, and 5 years were 42\% - 57\% [3] [4], 39\% $52 \%$ [3] [4], and $38 \%$ - 50\%, respectively [4], and in a nonrandomized trial of $90 \%$, after 2 years as well as after 5 years [5]. The own data showed locoregional control rates of $74.0 \%, 72.6 \%$, and $68.7 \%$ after 2,3 , and 5 years, respectively.

\subsection{Survival Rates}

The published survival rates of postoperative treated patients were $56 \%-71 \%$ after 2 years [1] [2] and 38\% - 53\% [1] [2] after 5 years, as well as after definitive treatment of $38 \%-60 \%$ after 2 years [3] [4], 26\% - 37\% after 3 years [3] [4], and $24 \%-29 \%$ after 5 years [4] as well as in a nonrandomized trial of $80 \%, 58 \%$, and $50 \%$ after 2,3 , and 5 years [5]. In our analysis, we found comparable survival rates of $71.8 \%$ and $46.6 \%$ after 2 years for the postoperative and definitive treated patients as well as $51.1 \%$ and $30.1 \%$ after 5 years.

Bernier et al. described a rate of second malignant diseases after 5 years of $12.5 \%$ [2]. We found 145 (15.5\%) second malignant diseases after a median follow-up of 21 months; 85 of these 145 (58.6\%) second malignant diseases led to patient death. This increased rate could also be an effect of the nonselected patient group.

In comparison to the published trials, we treated an unselected patient population. The median age of our patient group is an average of 5 years older. We treated a larger spectrum of primary tumour localisations, and 381 of 935 (41.7\%) patients had exclusion criteria of the mentioned radiochemotherapy trials.

\subsection{Causes of Death}

One reason for the short survival of these patients could be high comorbidity due to long-term alcohol and nicotine consumption, when $34 \%$ of the patients died from intercurrent diseases. These patients did not live longer than patients who died due to the failure of locoregional therapy or exclusively distant disease progression. Patients who survived longer had a high risk of suffering from a second malignant disease with a reduced prognosis.

\section{Conclusion}

The survival of patients with head and neck carcinoma after definitive or postoperative radio-chemotherapy was lower despite acceptable locoregional control rates of treatment.

\section{Conflict of Interest}

All authors declare no conflicts of interest.

\section{References}

[1] Cooper, J.S., Pajak, T.F., Forastiere, A.A., et al. (2004) Postoperative Concurrent 
Radiotherapy and Chemotherapy for High-Risk Squamous-Cell Carcinoma of the Head and Neck. The New England Journal of Medicine, 350, 1945-1952.

https://doi.org/10.1056/NEJMoa032646

[2] Bernier, J., Domenge, C., Ozsahin, M., et al. (2004) Postoperative Irradiation with or without Concomitant Chemotherapy for Locally Advanced Head and Neck Cancer. The New England Journal of Medicine, 350, 1937-1944.

https://doi.org/10.1056/NEJMoa032641

[3] Staar, S., Rudat, V., Stuetzer, H., et al. (2001) Intensified Hyperfractionated Accelerated Radiotherapy Limits the Additional Benefit of Simultaneous ChemotherapyResults of a Multicentric Randomized German Trial in Advanced Head-and-Neck Cancer. International Journal of Radiation Oncology • Biology • Physics, 50, 1161-1171. https://doi.org/10.1016/S0360-3016(01)01544-9

[4] Budach, V., Stuschke, M., Budach, W., et al. (2005) Hyperfractionated Accelerated Chemoradiation with Concurrent Fluorouracil-Mitomycin Is More Effective than Dose-Escalated Hyperfractionated Accelerated Radiation Therapy Alone in Locally Advanced Head and Neck Cancer: Final Results of the Radiotherapy Cooperative Clinical Trials Group of the German Cancer Society 95-06 Prospective Randomized Trial. Journal of Clinical Oncology, 23, 1125-1135.

https://doi.org/10.1200/JCO.2005.07.010

[5] Adelstein, D.J., Saxton, J.P., Lavertu, P., et al. (2002) Maximizing Local Control and Organ Preservation in Stage IV Squamous Cell Head and Neck Cancer with Hyperfractionated Radiation and Concurrent Chemotherapy. Journal of Clinical Oncology, 5, 1405-1410. https://doi.org/10.1200/JCO.2002.20.5.1405 